\title{
OPTIMAL CONTINGENT CLAIMS ${ }^{1}$
}

\author{
BY ANDRIUS JANKUNAS
}

Goldman, Sachs \& Co.

\begin{abstract}
Given a particular market variable, which could be finite dimensional (e.g., a price vector of a collection of stocks) or infinite dimensional (e.g., a price trajectory of some security over some period of time), we find the unique optimal European claim contingent on that variable in the sense that, for a given price and risk tolerance level, this claim has the highest expected return possible. The optimal contingent claims seem to be attractive investment instruments and are proposed for trade in derivatives markets.
\end{abstract}

1. Introduction. In recent years, derivatives markets achieved an extremely high level of flexibility. Derivative securities (or contingent claims) with virtually any contingency relation to their underlying assets can be purchased or synthesized from other derivatives. There is an extensive literature on how to price various derivative securities in the arbitrage-free manner under certain assumptions and market dynamics models; see, for example $[1,3,6,7]$. However, the problem of an optimal (in some sense) choice of a derivative security for an investment has not received as much attention. Consider an investor willing to invest $V$ dollars and having no hedging or other interests which would make him look for a derivative security with a particular payoff function on particular underlying assets. There is a huge choice of derivative securities with the same price $V$ but quite different properties, such as risk and expected return. Therefore, the investor is facing a rather difficult problem of choosing the best security in some sense for the given price $V$. The "good" properties of a security are high expected return and low risk. Since these properties clearly contradict each other, a natural investor's choice would be a derivative security with the highest expected return, price not exceeding $V$, and risk (measured in some particular way) not exceeding some tolerance level. Some kind of restriction on the risk is not only natural but also necessary for this problem to be meaningful because one can buy or synthesize a security with an arbitrarily high expected return (and also high risk) and zero price; see Remark 2.3.

In this paper we consider a special case of this problem when the investor has decided on a contingent claim of the European type paying $g(X)$ dollars at a maturity time $T$, where $X$ is an $\mathscr{X}$-valued market variable whose value is known by the maturity time $T,(\mathscr{X}, \mathscr{B})$ is a measurable space, and $g: \mathscr{X} \rightarrow$ $\mathbb{R}$ is a measurable contingency relation function. Typical examples of the

Received March 2000; revised November 2000.

${ }^{1}$ Written during author's visiting professorship at the University of Michigan.

AMS 2000 subject classifications. Primary 91B28; secondary 60G46.

Key words and phrases. Contingent claim, derivative security, equivalent martingale measure, payoff function. 
underlying market variable $X$ are a price of a particular stock or index at time $T(\mathscr{V}=[0, \infty))$, a vector of values of $n$ securities at times before $T\left(\mathscr{X}=\mathbb{R}^{n}\right)$, a trajectory of an $n$-dimensional price process observed by time $T\left(\mathscr{X}=\left(\mathbb{R}^{n}\right)^{[0, T]}\right.$ and $\mathscr{B}$ is the Borel sigma algebra with respect to the product topology), for example, Asian option. The problem consists of finding a measurable function $g_{V, Z}^{*}: \mathscr{X} \rightarrow \mathbb{R}$, maximizing the expected payoff $E g(X)$, over the set of all measurable functions $g: \mathscr{X} \rightarrow \mathbb{R}$ with property that the price of the corresponding contingent claim does not exceed $V$, and the risk measured by the payoff standard deviation $\sqrt{\operatorname{Var}(g(X))}$ does not exceed some tolerance level $Z \geq 0$. Under natural and usual assumptions in the literature, we show in this paper that such an optimal payoff function exists, is unique and find it in an explicit form. Since such an optimal contingent claim often can be synthesized from other derivatives already available in the market (see, e.g., [3], Chapter 8), this result can be viewed as an optimal design of a portfolio consisting of assets with the same underlying market variable. On the other hand, this result can be viewed as a proposal of a new class of contingent claims for trade in derivatives markets.

In Section 2, we derive the general result. In Section 3, we look into the structure of European contingent claims, and introduce the notion of an optimal forward contract. In Section 4, we consider the case when optimal contingent claims are not traded in derivatives markets, and construct an optimal portfolio from a finite number of derivative securities available in the market. In Sections 5 and 6, we present examples when $X$ is a stock price and a trajectory of a stock price, respectively. At the end of Section 6 , some comments on American options are also given.

2. General case. Let $\left(\Omega, \mathscr{F},\left\{\mathscr{F}_{t}\right\}_{0 \leq t \leq \mathscr{T}}, P\right)$ be a complete filtered probability space underlying all market variables under consideration, where the filtration $\left\{\mathscr{F}_{t}\right\}_{0 \leq t \leq \mathscr{T}}$ on $(\Omega, \mathscr{F})$ is such that $\mathscr{F}_{t}$ contains all events that happen on or before time $t \in[0, \mathscr{T}]$, and $\mathscr{T}<\infty$ is a sufficiently big time horizon beyond all transaction dates of our interest. Let $r_{t}, 0 \leq t \leq \mathscr{T}$, be the $\mathscr{T}_{t}$-adapted short rate process, and denote

$$
\beta_{t} \stackrel{\text { def }}{=} \exp \left(\int_{0}^{t} r_{s} d s\right)
$$

that is, $\beta_{t}$ is the unit rolled-up money market account. In this paper, we make the following assumption, usual in the literature of mathematical finance (see, e.g., $[1,3,6])$.

(A) There exists an equivalent martingale measure $\widetilde{P}$ over $(\Omega, \mathscr{F}$, $\left.\left\{\mathscr{F}_{t}\right\}_{0 \leq t \leq \mathscr{T}}, P\right)$ with respect to the numeraire $\beta_{t}$. In other words, there exists a probability measure $\widetilde{P}$ over $(\Omega, \mathscr{F})$, such that $\widetilde{P}$ and $P$ are equivalent, the density (Radon-Nikodym derivative) $\xi(\omega)=\frac{d \widetilde{P}}{d P}(\omega)$ is square-integrable with respect to $P$,

$$
E \xi^{2}=\int_{\Omega} \xi^{2}(\omega) d P(\omega)<\infty
$$


and for any $\mathscr{T}_{t}$-adaptive price process $Y_{t}$,

$$
\widetilde{E}\left(Y_{t} / \beta_{t} \mid \mathscr{F}_{s}\right)=Y_{s} / \beta_{s} \quad \forall 0 \leq s \leq t \leq \mathscr{T} .
$$

Here and further $\widetilde{E}$ and $E$ are expectations with respect to $\widetilde{P}$ and $P$, respectively.

This assumption implies that the price of a unit zero coupon bond with maturity $t$ at time zero is

$$
b_{t}=\widetilde{E} \beta_{t}^{-1} .
$$

Consider a measurable space $(\mathscr{X}, \mathscr{B})$ and an $\mathscr{T}_{T}$-measurable market variable $X: \Omega \rightarrow \mathscr{X}$, where $T \in[0, \mathscr{T}]$. Define $F: \mathscr{B} \rightarrow[0,1]$ to be the probability measure on $(\mathscr{X}, \mathscr{B})$ induced by $P$ and $X$; that is, $F(B)=P\left(X^{-1}(B)\right)$ for all $B \in \mathscr{B}$, and let $\widetilde{F}: \mathscr{B} \rightarrow[0,1]$ be the analogous probability measure induced by $\widetilde{P}$ and $X$. Obviously, the density

$$
\varphi(x) \stackrel{\text { def }}{=} \frac{d \widetilde{F}}{d F}(x)=E(\xi \mid X=x) .
$$

Let us consider a claim contingent on $X$ with payoff $g(X)$ at maturity $T$, where $g: \mathscr{X} \rightarrow \mathbb{R}$ is measurable. If the assumption (A) is fulfilled, then the value of this contingent claim at time zero is

$$
\begin{aligned}
v(g) & \stackrel{\text { def }}{=} \widetilde{E}\left(g(X) \beta_{T}^{-1}\right)=b_{T} \widetilde{E}\left(g(X) b_{T}^{-1} \beta_{T}^{-1}\right) \\
& =b_{T} \widehat{E} g(X)=b_{T} \int_{\mathscr{X}} g(x) d \widehat{F}(x),
\end{aligned}
$$

where $\widehat{E}$ is the expectation with respect to the measure $\widehat{P}$ on $(\Omega, \mathscr{F})$ which is absolutely continuous with respect to $P$, and the density

$$
\frac{d \widehat{P}}{d P}(\omega)=b_{T}^{-1} \beta_{T}^{-1}(\omega) \frac{d \widetilde{P}}{d P}(\omega)=b_{T}^{-1} \beta_{T}^{-1}(\omega) \xi(\omega) ;
$$

$\widehat{F}: \mathscr{B} \rightarrow[0,1]$ is the probability measure on $(\mathscr{X}, \mathscr{B})$ induced by $\widehat{P}$ and $X$. Obviously, $\widehat{F}$ is absolutely continuous with respect to $F$ and has the density

$$
\psi(x) \stackrel{\text { def }}{=} \frac{d \widehat{F}}{d F}(x)=b_{T}^{-1} \widetilde{E}\left(\beta_{T}^{-1} \mid X=x\right) \varphi(x) .
$$

REMARK 2.1. It is easy to check (see, e.g., [7], Section 7.5) that $\widehat{P}$ is an equivalent martingale measure over $\left(\Omega, \mathscr{F},\left\{\mathscr{F}_{t}\right\}_{0 \leq t \leq T}, P\right)$ with respect to the numeraire $\hat{\beta}_{t} \stackrel{\text { def }}{=} \widetilde{E}\left(\beta_{t} / \beta_{T} \mid \mathscr{F}_{t}\right)$, the price of a unit zero coupon bond with maturity $T$ at time $t$.

REMARK 2.2. In some important cases, $X$ and $\beta_{T}^{-1}$ are $\widetilde{P}$-independent. For example, if $X$ is a price of a stock or a trajectory of a stock price process, then it is common to assume that the short rate process $r_{t}$ is deterministic (see, e.g., $[1,3])$. In such a case, we have $\psi(x)=\varphi(x)$. 
The contingent claim with payoff $g(X)$ at maturity $T$ has the expected payoff (or return)

$$
p(g) \stackrel{\text { def }}{=} E g(X)=\int_{\mathscr{X}} g(x) d F(x)
$$

and the variance of the payoff

$$
z^{2}(g) \stackrel{\text { def }}{=} \operatorname{Var}(g(X))=\int_{\mathscr{X}} g^{2}(x) d F(x)-\left(\int_{\mathscr{X}} g(x) d F(x)\right)^{2} .
$$

Notation. For any two probability measures $\mu_{0}$ and $\mu_{1}$ on a measurable space $(\mathscr{X}, \mathscr{B})$, any numbers $\alpha \in \mathbb{R}$ and $\gamma \geq 0$, we denote by $G\left(\mu_{0}, \mu_{1}, \alpha, \gamma\right)$ the set of all measurable functions $g: \mathscr{X} \rightarrow \mathbb{R}$, such that

$$
\int_{\mathscr{X}} g(x) d \mu_{1}(x) \leq \alpha
$$

and

$$
\int_{\mathscr{X}} g^{2}(x) d \mu_{0}(x)-\left(\int_{\mathscr{X}} g(x) d \mu_{0}(x)\right)^{2} \leq \gamma^{2}
$$

In this paper, we consider the following problem. Given a price $V \in \mathbb{R}$ and a risk tolerance level $Z \geq 0$, we want to find a claim contingent on $X$ with as big expected payoff as possible. In other words, we want to find $g_{V, Z}^{*} \in$ $G\left(F, \widehat{F}, V / b_{T}, Z\right)$, such that $p\left(g_{V, Z}^{*}\right) \geq p(g)$ for all $g \in G\left(F, \widehat{F}, V / b_{T}, Z\right)$.

REMARK 2.3. From (2.6) and (2.9) we see that any $g \in L_{2}(F)$ orthogonal to $\psi$ and not orthogonal to 1 , with respect to the inner product in $L_{2}(F)$, can give rise to a contingent claim with an arbitrarily high expected return and zero price. For example, if we take $g_{n}(x)=n\left(\psi^{-1}(x)-1\right)$, then

$$
v\left(g_{n}\right)=b_{T}\left\langle g_{n}, \psi\right\rangle_{L_{2}(F)}=b_{T} \int_{\mathscr{X}} g_{n}(x) \psi(x) d F(x)=0
$$

and

$$
p\left(g_{n}\right)=\left\langle g_{n}, 1\right\rangle_{L_{2}(F)}=\left\langle g_{n}, \psi^{-1}\right\rangle_{L_{2}(\widehat{F})}=n \widehat{E}\left(\psi^{-1}(X)-1\right)^{2} \rightarrow \infty
$$

as $n \rightarrow \infty$. Therefore, some kind of restriction on the risk, such as $z(g) \leq Z$, of payoff functions under consideration is necessary to make our maximization problem meaningful.

The following lemma is the key to the solution of the problem.

LEMMA 2.4. Let $\mu_{0}$ and $\mu_{1}$ be two probability measures on a measurable space $(\mathscr{X}, \mathscr{B})$, such that $\mu_{1}$ is absolutely continuous with respect to $\mu_{0}$, and the Radon-Nikodym derivative $\zeta(x)=\frac{d \mu_{1}}{d \mu_{0}}(x)$ is square integrable with respect to $\mu_{0}$ :

$$
Q_{\mu_{0}, \mu_{1}} \stackrel{\text { def }}{=} \int_{\mathscr{X}} \zeta^{2}(x) d \mu_{0}(x)<\infty
$$


For any $\alpha \in \mathbb{R}$ and $\gamma \geq 0$ define

$$
g_{\alpha, \gamma}(x) \stackrel{\text { def }}{=} \alpha+\frac{\gamma}{\sqrt{Q_{\mu_{0}, \mu_{1}}-1}}\left[Q_{\mu_{0}, \mu_{1}}-\zeta(x)\right] .
$$

Then $g_{\alpha, \gamma} \in G\left(\mu_{0}, \mu_{1}, \alpha, \gamma\right)$, and

$$
\int_{\mathscr{X}} g(x) d \mu_{0}(x) \leq \int_{\mathscr{X}} g_{\alpha, \gamma}(x) d \mu_{0}(x)=\alpha+\gamma \sqrt{Q_{\mu_{0}, \mu_{1}}-1}
$$

for all $g \in G\left(\mu_{0}, \mu_{1}, \alpha, \gamma\right)$. Moreover, the inequality is strict unless $g=g_{\alpha, \gamma}$ almost sure with respect to $\mu_{0}$.

PROOF. By an elementary calculation one can show that

$$
\int_{\mathscr{X}} g_{\alpha, \gamma}(x) d \mu_{1}(x)=\alpha
$$

and

$$
\int_{\mathscr{X}} g_{\alpha, \gamma}^{2}(x) d \mu_{0}(x)-\left(\int_{\mathscr{X}} g_{\alpha, \gamma}(x) d \mu_{0}(x)\right)^{2}=\gamma^{2} .
$$

Therefore, $g_{\alpha, \gamma} \in G\left(\mu_{0}, \mu_{1}, \alpha, \gamma\right)$.

Now, let us fix some $g \in G\left(\mu_{0}, \mu_{1}, \alpha, \gamma\right)$. We can view $g$ and $\zeta$ as random variables on the probability space $\left(\mathscr{X}, \mathscr{B}, \mu_{0}\right)$. Obviously, $E \zeta=1, \operatorname{Var}(\zeta)=$ $Q_{\mu_{0}, \mu_{1}}-1, E(g \zeta) \leq \alpha$ and $\operatorname{Var}(g) \leq \gamma^{2}$. Therefore, by the Schwarz inequality,

$$
\begin{gathered}
\int_{\mathscr{X}} g_{\alpha, \gamma}(x) d \mu_{0}(x)-\int_{\mathscr{X}} g(x) d \mu_{0}(x)=\gamma \sqrt{Q_{\mu_{0}, \mu_{1}}-1}+\alpha-E g \\
\geq \sqrt{\operatorname{Var}(g) \operatorname{Var}(\zeta)}+\operatorname{Cov}(g, \zeta) \geq 0 .
\end{gathered}
$$

The first inequality is strict unless $\operatorname{Var}(g)=\gamma^{2}$ and $E(g \zeta)=\alpha$. The second inequality is strict unless $g=C-D \zeta$ almost sure for some $C \in \mathbb{R}$ and $D \geq 0$. Therefore, both inequalities in (2.14) turn into equalities if and only if $g=g_{\alpha, \gamma}$ almost sure with respect to $\mu_{0}$.

REMARK 2.5. The nonconstructive form of the Lemma 2.4 proof was chosen for it's simplicity, conciseness, and independence of any references to the infinite dimensional optimization literature. The reader could easily derive the same result in a constructive way using Lagrange multipliers in the corresponding infinite dimensional linear-quadratic optimization problem belonging to the theory of mean variance analysis.

Denote

$$
Q \stackrel{\text { def }}{=} Q_{F, \widehat{F}}=\int_{\mathscr{X}} \psi^{2}(x) d F(x)
$$


From (2.1) we see that $\beta_{t}^{-1} \in[0,1]$ for all $t \geq 0$. Therefore, by (2.8), (2.5) and assumption (A) we have

$$
\begin{aligned}
Q \leq b_{T}^{-2} E \varphi^{2}(X) & =b_{T}^{-2} E\left([E(\xi \mid X)]^{2}\right) \leq b_{T}^{-2} E\left(E\left(\xi^{2} \mid X\right)\right) \\
& =b_{T}^{-2} E \xi^{2}<\infty .
\end{aligned}
$$

The following theorem is the main result of this paper.

THEOREM 2.6. Suppose that assumption (A) holds. Given a price $V \in \mathbb{R}$ and a risk tolerance level $Z \geq 0$, define

$$
g_{V, Z}^{*}(x) \stackrel{\text { def }}{=} V / b_{T}+\frac{Z}{\sqrt{Q-1}}[Q-\psi(x)]
$$

Then $v\left(g_{V, Z}^{*}\right)=V, z\left(g_{V, Z}^{*}\right)=Z$ and

$$
p\left(g_{V, Z}^{*}\right)=V / b_{T}+Z \sqrt{Q-1} .
$$

Moreover, for any measurable payoff function $g: \mathscr{X} \rightarrow \mathbb{R}$ with price $v(g) \leq V$ and risk $z(g) \leq Z$, we have

$$
p(g) \leq p\left(g_{V, Z}^{*}\right)
$$

and the inequality is strict unless $g=g_{V, Z}^{*}$ almost sure with respect to $F$.

The theorem follows from (2.16) and Lemma 2.4 with $\alpha=V / b_{T}$ and $\gamma=Z$.

3. Optimal forward contracts. We call a derivative security a forward contract if it has zero price. Any European contingent claim with payoff $g(X)$ at maturity $T$ can be viewed as a portfolio consisting of $v(g) / b_{T}$ unit zero coupon bonds with maturity $T$ and a forward contract with payoff $q(X)=$ $g(X)-v(g) / b_{T}$ at maturity $T$. Therefore, price and risk can be separated, and one can adopt the view that European derivatives markets consist only of zero coupon bonds (nonzero price, zero risk) and forward contracts (zero price, nonzero risk). Thus, the problem considered in Section 2 is equivalent to finding a forward contract payoff function $q^{*}: \mathscr{X} \rightarrow \mathbb{R}, v\left(q^{*}\right)=0$, with unit risk $\left(z\left(q^{*}\right)=1\right)$ and maximal expected return; that is,

$$
p\left(q^{*}\right) \geq p(q) \quad \forall q: \mathscr{X} \rightarrow \mathbb{R}: v(q)=0, z(q)=1 .
$$

By Theorem 2.6,

$$
q^{*}(x)=\frac{Q-\psi(x)}{\sqrt{Q-1}}
$$

and

$$
p\left(q^{*}\right)=\sqrt{Q-1}
$$

For any $\gamma>0$, the forward contract payoff function $\gamma q^{*}$ is optimal in the sense that it provides most expected return per unit of risk. 
Given a price $V \in \mathbb{R}$ and risk tolerance level $Z \geq 0$, we have

$$
g_{V, Z}^{*}(x)=V / b_{T}+Z q^{*}(x)
$$

that is, the optimal contingent claim can be synthesized by investing $V$ dollars into zero coupon bonds with maturity $T$ and entering into $Z$ optimal unit forward contracts defined by (3.1). Thus, in order to make optimal contingent claims available in the market, it is not necessary to trade them for every price $V$ and risk tolerance level $Z$. Trading of optimal forward contracts is sufficient.

For any measurable payoff function $g: \mathscr{X} \rightarrow \mathbb{R}, z(g)>0$, define

$$
R(g) \stackrel{\text { def }}{=} \frac{p(g)-v(g) / b_{T}}{z(g) \sqrt{Q-1}} .
$$

Obviously, $R(g)=p(q) / p\left(q^{*}\right)$, where $q$ is the unit forward contract corresponding to $g$. Therefore, $R\left(g_{V, Z}^{*}\right)=1$ for any $V \in \mathbb{R}, Z>0$, and $R(g) \in$ $[-1,1)$ if $g$ is not optimal for any price and risk. The criterion $R(\cdot)$ can be used as a performance measure of contingent claims.

4. Optimal finite portfolios. For any given price and risk tolerance level, Theorem 2.6 provides the most attractive claim contingent on the market variable $X$. If this claim or optimal forward contract $q^{*}$ were traded in derivatives market, it would be a natural investor's choice. However, usually only a finite set of more traditional contingent claims is available. In such a case, an investor can approximate the optimal contingent claim by solving a finite dimensional optimization problem of finding an optimal portfolio of the available contingent claims. Let $h_{i}: \mathscr{X} \rightarrow \mathbb{R}, i=1, \ldots, n$, be measurable payoff functions of available in the market contingent claims with maturity $T$. Then one can construct a portfolio consisting of $c$ zero coupon bonds and $a_{i}$ contingent claims with index $i, i=1, \ldots, n$. The payoff function of such a portfolio is

$$
g(x \mid c, a)=c+\sum_{i=1}^{n} a_{i} h_{i}(x)=c+h^{\top}(x) a
$$

where $a \stackrel{\text { def }}{=}\left(a_{1}, \ldots, a_{n}\right)^{\top}$, and $h(x) \stackrel{\text { def }}{=}\left(h_{1}(x), \ldots, h_{n}(x)\right)^{\top}$. Denote

$$
\begin{gathered}
H \stackrel{\text { def }}{=} E h(X), \quad \widehat{H} \stackrel{\text { def }}{=} \widehat{E} h(X), \\
M \stackrel{\text { def }}{=} \operatorname{Cov}(h(X), h(X))=E\left[h(X) h^{\top}(X)\right]-H H^{\top} .
\end{gathered}
$$

Obviously,

$$
\begin{gathered}
v(c, a) \stackrel{\text { def }}{=} v(g(\cdot \mid c, a))=b_{T}\left(c+\widehat{H}^{\top} a\right), \\
p(c, a) \stackrel{\text { def }}{=} p(g(\cdot \mid c, a))=c+H^{\top} a
\end{gathered}
$$


and

$$
z^{2}(c, a) \stackrel{\text { def }}{=} z^{2}(g(\cdot \mid c, a))=a^{\top} M a .
$$

Now let us formulate an analog of Theorem 2.6 for finite portfolios.

THEOREM 4.1. Suppose that assumption (A) holds, and the matrix $M$ is positive definite. Given a price $V \in \mathbb{R}$ and a risk tolerance level $Z \geq 0$, define

$$
\begin{gathered}
a_{V, Z}^{*} \stackrel{\text { def }}{=} Z \frac{M^{-1}(H-\widehat{H})}{\sqrt{(H-\widehat{H})^{\top} M^{-1}(H-\widehat{H})}}, \\
c_{V, Z}^{*} \stackrel{\text { def }}{=} V / b_{T}-\widehat{H}^{\top} a_{V, Z}^{*} .
\end{gathered}
$$

Then $v\left(c_{V, Z}^{*}, a_{V, Z}^{*}\right)=V, z\left(c_{V, Z}^{*}, a_{V, Z}^{*}\right)=Z$ and

$$
p\left(c_{V, Z}^{*}, a_{V, Z}^{*}\right)=V / b_{T}+Z \sqrt{(H-\widehat{H})^{\top} M^{-1}(H-\widehat{H})} .
$$

Moreover, for any portfolio $(c, a) \neq\left(c_{V, Z}^{*}, a_{V, Z}^{*}\right)$ with price $v(c, a) \leq V$ and risk $z(c, a) \leq Z$, we have

$$
p(c, a)<p\left(c_{V, Z}^{*}, a_{V, Z}^{*}\right) .
$$

Proof. From (4.3) and (4.5), one easily gets $v\left(c_{V, Z}^{*}, a_{V, Z}^{*}\right)=V, z\left(c_{V, Z}^{*}\right.$, $\left.a_{V, Z}^{*}\right)=Z$ and $p\left(c_{V, Z}^{*}, a_{V, Z}^{*}\right)=V / b_{T}+Z \sqrt{(H-\widehat{H})^{\top} M^{-1}(H-\widehat{H})}$.

Now, let us fix some portfolio $(c, a) \in \mathbb{R} \times \mathbb{R}^{n}$ with price $v(c, a) \leq V$ and risk $z(c, a) \leq Z$. Obviously,

$$
\begin{aligned}
& p\left(c_{V, Z}^{*}, a_{V, Z}^{*}\right)-p(c, a) \\
& \geq \sqrt{a^{\top} M a} \sqrt{(H-\widehat{H})^{\top} M^{-1}(H-\widehat{H})}-(H-\widehat{H})^{\top} a \\
& \geq a^{\top} M^{1 / 2} M^{-1 / 2}(H-\widehat{H})-(H-\widehat{H})^{\top} a=0 .
\end{aligned}
$$

The first inequality is strict unless $v(c, a)=V$ and $z(c, a)=Z$. The second inequality follows from the Schwarz inequality for the inner product in Euclidean space and is strict unless $a=\gamma M^{-1}(H-\widehat{H})$ for some $\gamma \geq 0$. Therefore, both inequalities in (4.7) turn into equalities if and only if $c=c_{V, Z}^{*}$ and $a=a_{V, Z}^{*}$.

REMARK 4.2. For any $u \in \mathbb{R}^{n} \backslash\{0\}$, we have $u^{\top} M u=\operatorname{Var}\left(u^{\top} h(X)\right)$. Thus, $M$ is positive definite unless there exist $u \in \mathbb{R}^{n} \backslash\{0\}$ and $\gamma \in \mathbb{R}$, such that $u^{\top} h(x)=\gamma$ almost everywhere with respect to the measure $F$. 
REMARK 4.3. Let us fix some price $V \in \mathbb{R}$ and risk tolerance level $Z \geq 0$. By an elementary calculation one can show that for any measurable $g: \mathscr{X} \rightarrow \mathbb{R}$ with price $v(g)=V$ and risk $z(g)=Z$,

$$
\begin{aligned}
\left\|g_{V, Z}^{*}-g\right\|_{L_{2}(F)}^{2} & =\int_{\mathscr{X}}\left(g_{V, Z}^{*}(x)-g(x)\right)^{2} d F(x) \\
& =\varepsilon_{V, Z}^{2}(g)+\frac{2 Z}{\sqrt{Q-1}} \varepsilon_{V, Z}(g),
\end{aligned}
$$

where $\varepsilon_{V, Z}(g) \stackrel{\text { def }}{=} p\left(g_{V, Z}^{*}\right)-p(g) \geq 0$ is the expected return deficiency of $g$ as compared to the optimal claim $g_{V, Z}^{*}$ given by Theorem 2.6. In particular,

$$
\varepsilon_{V, Z}\left(g\left(\cdot \mid c_{V, Z}^{*}, a_{V, Z}^{*}\right)\right)=Z\left(\sqrt{Q-1}-\sqrt{(H-\widehat{H})^{\top} M^{-1}(H-\widehat{H})}\right) .
$$

From (4.8) it follows that

$$
\left(c_{V, Z}^{*}, a_{V, Z}^{*}\right)=\arg \min _{(c, a): v(c, a)=V, z(c, a)=Z}\left\|g_{V, Z}^{*}-g(\cdot \mid c, a)\right\|_{L_{2}(F)} ;
$$

that is, the maximum expected return is achieved by the portfolio with price $V$ and risk $Z$ whose payoff function is closest to $g_{V, Z}^{*}$ in $L_{2}(F)$ space.

5. Optimal claim contingent on a stock price. In this section, we consider an example when $\mathscr{X}=[0, \infty), \mathscr{B}$ is the Borel sigma algebra and $X=S_{T}$, where $S_{t}, 0 \leq t \leq T$, is a price process of some no dividend paying stock described by the Black-Scholes stochastic differential equation (see, e.g. $[1,3])$ in the sense of Itô:

$$
d S_{t}=\mu_{t} S_{t} d t+\sigma_{t} S_{t} d W_{t}
$$

where $W_{t}$ is a standard Brownian motion on $\left(\Omega, \mathscr{F},\left\{\mathscr{F}_{t}\right\}_{0 \leq t \leq T}, P\right)$. The growth coefficient $\mu_{t}$, volatility $\sigma_{t} \neq 0$ and the short rate process $r_{t}$ are assumed to be deterministic. Without a loss of generality we may assume that $S_{0}=1$. It is well known (see $[3,1]$ ) that for this Black-Scholes model there exists an equivalent martingale measure $\widetilde{P}$, that is, condition (A) is fulfilled, and

$$
d S_{t}=r_{t} S_{t} d t+\sigma_{t} S_{t} d \widetilde{W}_{t}
$$

where $\widetilde{W}_{t}=\int_{0}^{t}\left(\mu_{s}-r_{s}\right) \sigma_{s}^{-1} d s+W_{t}$ is a standard Brownian motion on $(\Omega, \mathscr{F}$, $\left.\left\{\mathscr{F}_{t}\right\}_{0 \leq t \leq T}, \widetilde{P}\right)$. Moreover, it is well known (see, e.g., $[3,1]$ ) that in this case both distributions $F$ and $\widetilde{F}$ are lognormal, and their densities, with respect to the Lebesgue measure over $[0, \infty)$, are

$$
f(x)=\frac{1}{\sqrt{2 \pi T} \sigma x} \exp \left\{-\frac{1}{2 T \sigma^{2}}\left[\ln x-\left(\mu-\frac{\sigma^{2}}{2}\right) T\right]^{2}\right\}
$$

and

$$
\tilde{f}(x)=\frac{1}{\sqrt{2 \pi T} \sigma x} \exp \left\{-\frac{1}{2 T \sigma^{2}}\left[\ln x-\left(r-\frac{\sigma^{2}}{2}\right) T\right]^{2}\right\}
$$


respectively, where

$$
\mu=\frac{1}{T} \int_{0}^{T} \mu_{t} d t, \quad r=\frac{1}{T} \int_{0}^{T} r_{t} d t \quad \text { and } \quad \sigma^{2}=\frac{1}{T} \int_{0}^{T} \sigma_{t}^{2} d t .
$$

By Remark 2.2, we have

$$
\psi(x)=\varphi(x)=\frac{\tilde{f}(x)}{f(x)}=x^{-(\mu-r) / \sigma^{2}} e^{\frac{T}{\sigma^{2}}(\mu-r)\left(\mu+r-\sigma^{2}\right)},
$$

and Theorem 2.6 implies that, given a price $V \in \mathbb{R}$ and a risk tolerance level $Z \geq 0$, the maximum expected return is achieved by the payoff function

$$
g_{V, Z}^{*}(x)=V e^{r T}+\frac{Z}{\sqrt{Q-1}}\left[Q-\frac{\tilde{f}(x)}{f(x)}\right]=C_{1}-C_{2} x^{-(\mu-r) / \sigma^{2}},
$$

where

$$
\begin{gathered}
Q=\int_{0}^{\infty} \frac{\tilde{f}^{2}(x)}{f(x)} d x=e^{T(\mu-r)^{2} / \sigma^{2}} \\
C_{1}=V e^{r T}+\frac{Z Q}{\sqrt{Q-1}} \quad \text { and } \quad C_{2}=\frac{Z}{\sqrt{Q-1}} e^{\frac{T}{2 \sigma^{2}}(\mu-r)\left(\mu+r-\sigma^{2}\right)},
\end{gathered}
$$

and the maximal expected return $p\left(g_{V, Z}^{*}\right)=V e^{r T}+Z \sqrt{Q-1}$.

One might be interested in comparing the expected return of a popular in the market contingent claim, such as put or call option, to the expected return of the optimal contingent claim with the same price and risk. Denote by $h_{c, K}(x)=\max \{x-K, 0\}$ and $h_{p, K}(x)=\max \{K-x, 0\}$ the payoff functions of the European call and put options, respectively, with a strike price $K>0$. The relative to the optimal claim performance of these options can be measured by the payoff ratios $P R\left(h_{i, K}\right)=p\left(h_{i, K}\right) / p\left(g_{v\left(h_{i, K}^{*}\right), z\left(h_{i, K}\right)}\right) \in[0,1] ; i=c, p$. In Figure 1, the payoff ratios $P R\left(h_{c, K}\right)$ and $P R\left(h_{p, K}\right)$ are drawn as functions of

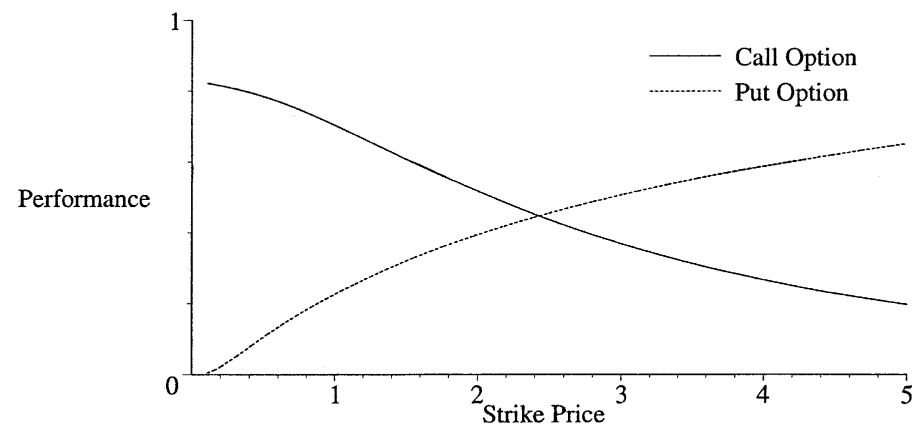

FIG. 1. Relative performance of the European call and put options measured by their expected return divided by the expected return of the optimal contingent claim with the same price and risk. Here $T=5, r=0.06, \mu=0.15$ and $\sigma=0.3$. 


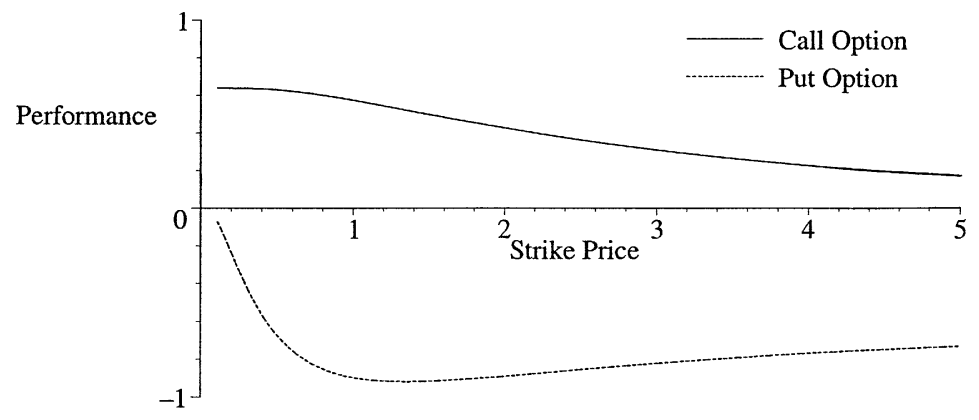

FIG. 2. Performance of the European call and put options measured by the criterion $R(\cdot)$ defined in (3.2). Here $T=5, r=0.06, \mu=0.15$ and $\sigma=0.3$.

the strike price $K$ for particular values of $T, r, \mu$ and $\sigma$. In view of Section 3, the drawback of $P R(\cdot)$ as a performance measure is the confounding of returns generated by the risk free (zero coupon bond) and risky (forward contract) parts of a contingent claim. This is the reason why $P R\left(h_{p, K}\right)$ is close to 1 for big values of $K$. Since the essential part of a European contingent claim is the corresponding forward contract, it seems that $R(\cdot)$ is the most natural measure of claim performance. In Figure 2, the performance values $R\left(h_{c, K}\right)$ and $R\left(h_{p, K}\right)$ are drawn as functions of the strike price $K$.

The optimal payoff function $g_{V, Z}^{*}$ might seem somewhat "scary" because $\lim _{x \rightarrow+0} g_{V, Z}^{*}(x)=-\infty$; that is, if something went absolutely wrong with the underlying stock, the investor would owe a huge amount of money. However, since $\lim _{x \rightarrow+0} g_{V, Z}^{*}(x) f(x)=\lim _{x \rightarrow+0} g_{V, Z}^{*}(x) \tilde{f}(x)=\lim _{x \rightarrow+0}\left[g_{V, Z}^{*}(x)\right]^{2} f(x)=0$, the truncated payoff function $\bar{g}_{V, Z, M}(x)=\max \left\{-M, g_{V, Z}^{*}(x)\right\}$ with a sufficiently large $M>0$ yields approximately the same price, risk and expected return as the optimal function $g_{V, Z}^{*}$, and therefore can be used instead.

REMARK 5.1. In order to calculate the optimal contingent claim defined by (5.2), one needs to know the parameters $\mu, r$ and $\sigma^{2}$. The parameters $r$ and $\sigma^{2}$ can be calculated (implied) from the current bond and call, put, or other option prices in the market. The drift coefficient $\mu$, corresponding to the "real world" probability measure $F$, cannot be implied from the current market data because the measure $\widehat{F}$ and prices of derivative securities do not depend on it. If one assumes that $\mu_{t}$ is approximately constant over time $\left(\mu_{t} \equiv \mu\right)$, one can estimate it from the historical data (see $[4,5]$ for results on asymptotically efficient estimation of drift parameters of multidimensional linear stochastic differential or difference equations which are also applicable to a more general case when $X$ is a price vector corresponding to several stocks). If one is reluctant to assume that stock behavior in the past is similar to its behavior in the future, one has to make an "educated" guess of $\mu$ expressing ones belief in the future growth of the underlying stock price. Therefore, optimal claims corresponding to different investors could be different. 
Now, consider a market where the only traded claims contingent on $X=S_{T}$ with maturity $T$ are call options with strike prices $K_{i}=\Delta(i-1), i=1, \ldots, n$, $\Delta>0$. So, the available contingent claims have payoff functions

$$
h_{i}(x)=\max \{x-\Delta(i-1), 0\}, \quad i=1, \ldots, n .
$$

Since $K_{0}=0$, the first call option is just the stock itself.

By an elementary integration from (4.1) and (4.2) we get

$$
\begin{aligned}
H_{i}= & E h_{i}(X)=e^{\mu T} \Phi\left(d_{\mu, K_{i}}+\sigma \sqrt{T}\right)-K_{i} \Phi\left(d_{\mu, K_{i}}\right), \\
\widehat{H}_{i}= & \widetilde{E} h_{i}(X)=e^{r T} \Phi\left(d_{r, K_{i}}+\sigma \sqrt{T}\right)-K_{i} \Phi\left(d_{r, K_{i}}\right), \\
M_{i j}=M_{j i}= & \operatorname{Cov}\left(h_{i}(X), h_{j}(X)\right) \\
= & e^{\left(2 \mu+\sigma^{2}\right) T} \Phi\left(d_{\mu, K_{j}}+2 \sigma \sqrt{T}\right)-2 K_{j} e^{\mu T} \Phi\left(d_{\mu, K_{j}}+\sigma \sqrt{T}\right) \\
& +K_{j}^{2} \Phi\left(d_{\mu, K_{j}}\right)+\left(K_{j}-K_{i}-H_{i}\right) H_{j},
\end{aligned}
$$

where $1 \leq i \leq j \leq n, d_{u, K} \stackrel{\text { def }}{=}\left[\left(u-\sigma^{2} / 2\right) T-\ln K\right] /(\sigma \sqrt{T})$ and $\Phi(\cdot)$ is the cumulative standard Normal distribution function (in particular, $d_{u, 0}=\infty$ and $\Phi(\infty)=1$ ). By Remark 4.2, the $n \times n$ matrix $M$ is positive definite, and Theorem 2.6 is valid.

In Figure 3, the payoff functions $g\left(\cdot \mid c_{0,1}^{*}, a_{0,1}^{*}\right)$ of optimal portfolios corresponding to various values of $\Delta$ and $n$ are drawn and compared to the optimal payoff function $g_{0,1}^{*}=q^{*}$ for particular choice of $T, r, \mu$ and $\sigma$. It is well known (see, e.g., [3], Chapter 8) that, if European call options expiring at $T$ were available with every strike price, then any payoff function could be replicated by a portfolio of these options. Therefore, in view of Remark 4.3, it is clear that for small values of $\Delta$ and big values of $n \Delta$ the optimal portfolio payoff function $g\left(\cdot \mid c_{0,1}^{*}, a_{0,1}^{*}\right)$ must be close to $q^{*}$, and the expected payoff $p\left(c_{0,1}^{*}, a_{0,1}^{*}\right)$ must be almost as high as $p\left(q^{*}\right)=\sqrt{Q-1}$.

6. Optimal claim contingent on a price trajectory. In this section, we consider the same stock price process $S_{t}, 0 \leq t \leq T$, as in Section 5. However, the claims we consider here are contingent not only on the maturity value $S_{T}$ but on the whole trajectory $S^{(T)}=\left(S_{t}, 0 \leq t \leq T\right)$. So, $\mathscr{X}=[0, \infty)^{[0, T]}$, and $\mathscr{B}$ is the Borel sigma algebra with respect to the product topology. Obviously, the set of all measurable functions $\{g: \mathscr{X} \rightarrow \mathbb{R}\}$ contains the set of all functions depending only on the last point of the trajectory $\left\{g: \mathscr{X} \rightarrow \mathbb{R} \mid g(x)=g_{0}(x(T))\right.$ for some measurable $\left.g_{0}:[0, \infty) \rightarrow \mathbb{R}\right\}$. Therefore, given a price $V$ and a risk tolerance level $Z$, the maximal expected return based on the whole trajectory must be greater or equal to the one based only on the maturity value of the price process. It is well known (see, e.g., [2], Section 3.12) that for any 

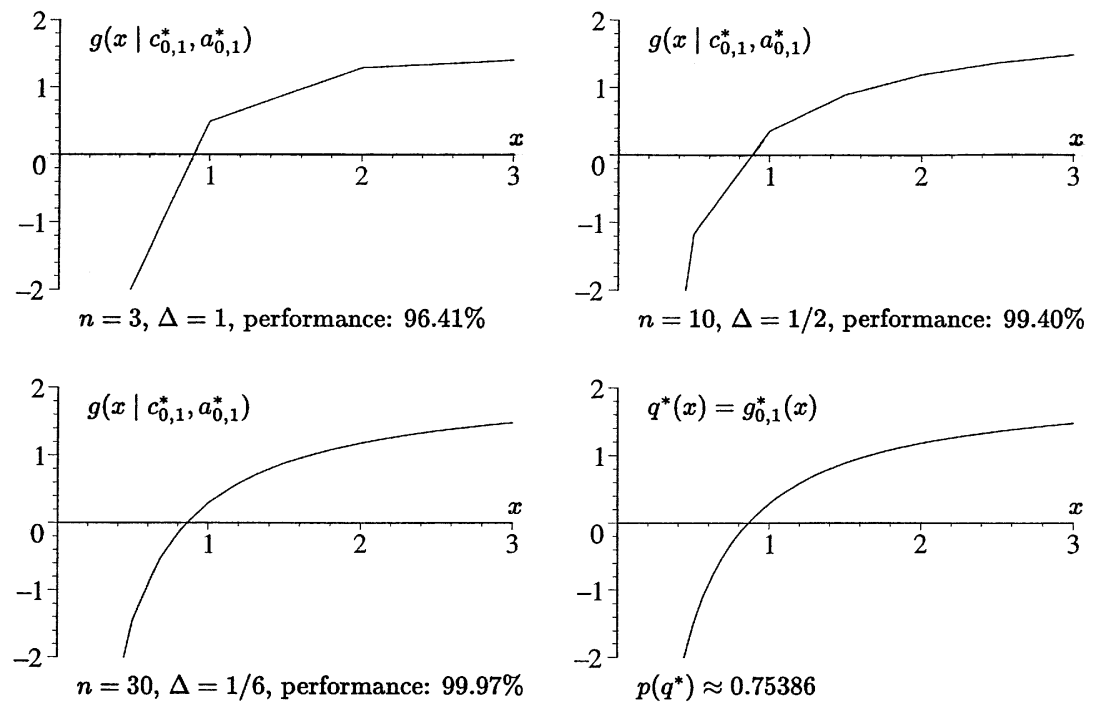

FIG. 3. Payoff functions $g\left(\cdot \mid c_{0,1}^{*}, a_{0,1}^{*}\right)$ of optimal portfolios corresponding to various values of $\Delta$ and $n$ compared to the optimal payoff function $q^{*}=g_{0,1}^{*}$. The performance of each portfolio is measured by $R\left(g\left(\cdot \mid c_{0,1}^{*}, a_{0,1}^{*}\right)\right)=p\left(c_{0,1}^{*}, a_{0,1}^{*}\right) / p\left(q^{*}\right)$. Here $T=5, r=0.06, \mu=0.15$ and $\sigma=0.3$.

$x \in \operatorname{Supp}(F)=\operatorname{Supp}(\widetilde{F}) \subseteq[0, \infty)^{[0, T]}=\mathscr{X}$ the density

$$
\begin{aligned}
\psi(x) & =\varphi(x)=\frac{d \tilde{F}}{d F}(x)=\exp \left\{\frac{1}{2} \int_{0}^{T} \frac{\mu_{t}^{2}-r_{t}^{2}}{\sigma_{t}^{2}} d t-\int_{0}^{T} \frac{\mu_{t}-r_{t}}{\sigma_{t}^{2}} \frac{d x_{t}}{x_{t}}\right\} \\
& =\exp \left\{\frac{1}{2} \int_{0}^{T} \frac{\mu_{t}-r_{t}}{\sigma_{t}^{2}}\left(\mu_{t}+r_{t}-\sigma_{t}^{2}\right) d t-\int_{0}^{T} \frac{\mu_{t}-r_{t}}{\sigma_{t}^{2}} d \ln x_{t}\right\}
\end{aligned}
$$

where the last equality follows from the Itô formula and the fact that $x \in$ $\operatorname{Supp}(F)$.

Theorem 2.6 implies that, given a price $V \in \mathbb{R}$ and a risk tolerance level $Z \geq 0$, the maximum expected payoff is achieved by the payoff function

$$
\begin{aligned}
g_{V, Z}^{*}(x) & =V e^{r T}+\frac{Z}{\sqrt{Q-1}}[Q-\varphi(x)] \\
& =C_{1}-C_{2} \exp \left\{-\int_{0}^{T} \frac{\mu_{t}-r_{t}}{\sigma_{t}^{2}} d \ln x_{t}\right\},
\end{aligned}
$$

where

$$
\begin{gathered}
Q=E \varphi^{2}\left(S^{(T)}\right)=\exp \left\{\int_{0}^{T}\left(\frac{\mu_{t}-r_{t}}{\sigma_{t}}\right)^{2} d t\right\} \\
C_{1}=V e^{r T}+\frac{Z Q}{\sqrt{Q-1}} \text { and } C_{2}=\frac{Z}{\sqrt{Q-1}} \exp \left\{\frac{1}{2} \int_{0}^{T} \frac{\mu_{t}-r_{t}}{\sigma_{t}^{2}}\left(\mu_{t}+r_{t}-\sigma_{t}^{2}\right) d t\right\}
\end{gathered}
$$


and the maximal expected payoff $p\left(g_{V, Z}^{*}\right)=V e^{r T}+Z \sqrt{Q-1}$. By the Schwarz inequality,

$$
\int_{0}^{T}\left(\frac{\mu_{t}-r_{t}}{\sigma_{t}}\right)^{2} d t \geq T\left(\frac{\mu-r}{\sigma}\right)^{2}
$$

where $\mu, r$, and $\sigma$ are defined in (5.1). Inequality (6.2) is strict, and therefore the expected payoff $p\left(g_{V, Z}^{*}\right)$ is higher than the one received in Section 5 , unless

$$
\mu_{t}-r_{t}=\alpha \sigma_{t}^{2}
$$

for some $\alpha \in \mathbb{R}$ almost everywhere with respect to the Lebesgue measure over $[0, T]$. If (6.3) holds (this happens when $\mu_{t} \equiv \mu, r_{t} \equiv r$ and $\sigma_{t}^{2} \equiv \sigma^{2}$, e.g.), then $g_{V, Z}^{*}(x)=C_{1}-C_{2} x_{T}^{-(\mu-r) / \sigma^{2}}$, and we return to the situation of Section 5 . In other words, if and only if (6.3) holds, then the optimal claim is contingent on the stock price process trajectory only through the stock price at maturity (the rest of the trajectory is disregarded).

Another peculiar consequence of (6.3) is that European contingent claims are preferable to American ones. Indeed, consider an American option paying $g\left(t, S_{t}\right)$ dollars if exercised at time $t \leq T$, where $g:[0, T] \times[0, \infty) \rightarrow \mathbb{R}$ is a measurable function. It is well known (see, e.g. $[1,6]$ ) that the price of this option is

$$
v_{a}(g)=\widetilde{E}\left(\beta_{\tau}^{-1} g\left(\tau, S_{\tau}\right)\right)=b_{T} \widehat{E}\left(\beta_{T} \beta_{\tau}^{-1} g\left(\tau, S_{\tau}\right)\right),
$$

where $\tau$ is the stopping time corresponding to the optimal exercise policy. We can view this American option as a European contingent claim paying $\beta_{T} \beta_{\tau}^{-1} g\left(\tau, S_{\tau}\right)$ at maturity $T$. The stopping time $\tau$ is $\mathscr{F}_{T}$-measurable, and can be viewed as a measurable function of the whole trajectory, $\tau=\tau\left(S^{(T)}\right)$. Since $\beta_{t}$ is deterministic, we can define a function $g_{a}: \mathscr{X}=[0, \infty)^{[0, T]} \rightarrow \mathbb{R}$ by

$$
g_{a}(x)=\beta_{T} \beta_{\tau(x)}^{-1} g\left(\tau(x), x_{\tau(x)}\right) .
$$

Theorem 2.6 implies that

$$
p\left(g_{a}\right)<p\left(g_{v\left(g_{a}\right), z\left(g_{a}\right)}^{*}\right),
$$

where $g_{v, z}$ is defined in (6.1). So, an American option on a stock is less attractive than the optimal European claim contingent on the whole stock price trajectory and having the same price and risk. Even more, if (6.3) holds, then from the previous discussion it follows that (6.4) also holds for $g_{v, z}^{*}$ defined in (5.2); that is, the optimal European claim contingent on a stock value at maturity $T$ is preferable not only to any other European claim, but also to any American option with the same price, risk and maturity.

REMARK 6.1. Even if the short rate process $r_{t}$ is stochastic, any American contingent claim, with maturity $T$, underlying price process $Y_{t} \in \mathscr{Y}, 0 \leq$ $t \leq T$, measurable payoff function $g:[0, T] \times \mathscr{Y} \rightarrow \mathbb{R}\left(\right.$ here $\left(\mathscr{Y}, \mathscr{B}_{\mathscr{Y}}\right)$ is some measurable space), and optimal exercise stopping time $\tau=\tau\left(Y^{(T)}, \beta^{(T)}\right)$, can 
be viewed as a European claim contingent on the extended market variable $\left(Y^{(T)}, \beta^{(T)}\right)$ with payoff

$$
g_{a}\left(Y^{(T)}, \beta^{(T)}\right)=\beta_{T} \beta_{\tau\left(Y^{(T)}, \beta^{(T)}\right)}^{-1} g\left(\tau\left(Y^{(T)}, \beta^{(T)}\right), Y_{\tau\left(Y^{(T)}, \beta^{(T)}\right)}\right)
$$

at maturity $T$, and, therefore, is inferior to the optimal contingent claim given by Theorem 2.6 with $\mathscr{X}=\left(Y^{(T)}, \beta^{(T)}\right)$.

\section{REFERENCES}

[1] Duffie, D. (1992). Dynamic Asset Pricing Theory. Princeton Univ. Press.

[2] Gikhman, I. I. and Skorokhod, A. V. (1972). Stochastic Differential Equations. Springer, Berlin.

[3] Hull, J. C. (1989). Options, Futures, and Other Derivative Securities. Prentice-Hall, Englewood Cliffs, NJ.

[4] JANKUNAS, A. and KHASMINSKII, R. Z. (1997). Estimation of parameters of linear homogeneous stochastic differential equations. Stochastic Process. Appl. 72 205-219.

[5] JANKunAS, A. and KHASMinskit, R. Z. (1998). Estimation of parameters of linear stochastic difference equations. Math. Methods Statist. 7 339-352.

[6] Karatzas, I. and Shreve, S. E. (1998). Methods of Mathematical Finance. Springer, New York.

[7] Rebonato, R. (1996). Interest-Rate Option Models. Wiley, New York.

GOLDMAN, SACHS \& Co.

85 BROAD STREET

NEW YORK, NY 10004

E-MAIL: andrius.jankunas@gs.com 